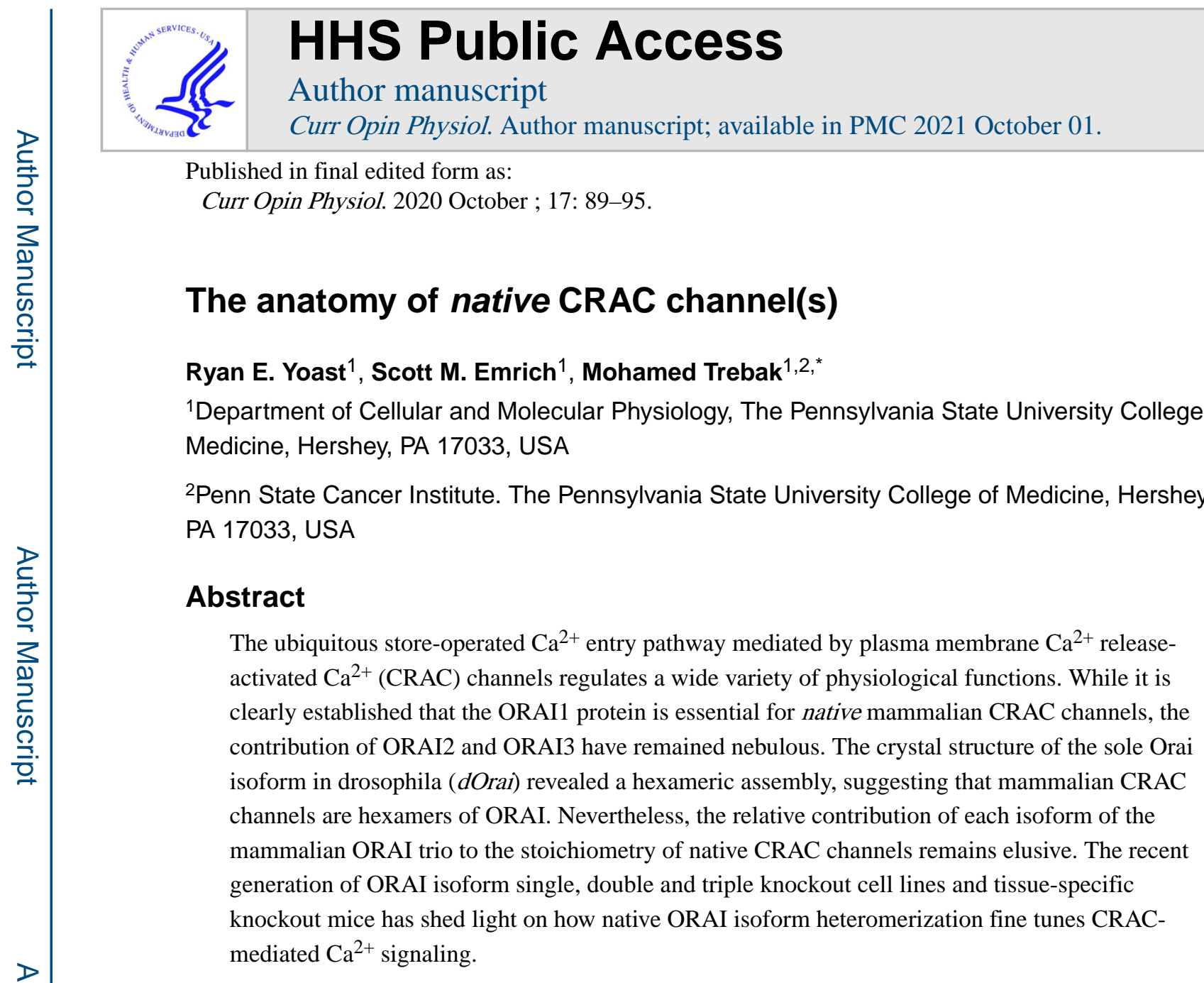

\title{
Keywords
}

ORAI; STIM; Calcium signaling; Store-operated calcium entry; CRAC channels

\section{Store-operated $\mathrm{Ca}^{2+}$ entry (SOCE)}

Physiological calcium $\left(\mathrm{Ca}^{2+}\right)$ concentrations in the extracellular space $(1-2 \mathrm{mM})$ and in the lumen of the endoplasmic reticulum (ER; $300 \mu \mathrm{M}-1 \mathrm{mM}$ ) are orders of magnitude higher than in the cytosol (50-100nM)[1]. Cytosolic $\mathrm{Ca}^{2+}$ concentrations are tightly regulated by means of channels, pumps, and transporters located on the surface of the plasma membrane (PM) and internal organelles. This tight control ensures efficient agonist mediated $\mathrm{Ca}^{2+}$ signaling while protecting from toxicity of elevated levels of cytosolic $\mathrm{Ca}^{2+[1]}$. Stimulation of cells with agonists to G-protein coupled receptors or receptor tyrosine kinases that couple to

“Correspondence: mtrebak@psu.edu.

Author contributions

R.E.Y., S.M.E. and M.T. contributed to the writing of the manuscript.

Publisher's Disclaimer: This is a PDF file of an unedited manuscript that has been accepted for publication. As a service to our customers we are providing this early version of the manuscript. The manuscript will undergo copyediting, typesetting, and review of the resulting proof before it is published in its final form. Please note that during the production process errors may be discovered which could affect the content, and all legal disclaimers that apply to the journal pertain.

CONFLICT OF INTEREST STATEMENT

The authors declare no competing interests. 
isoforms of the phosphoinositide-specific phospholipase C (PLC) cause the release of luminal $\mathrm{ER} \mathrm{Ca}^{2+}$ into the cytosol through ER-resident inositol 1,4,5-trisphosphate $\left(\mathrm{IP}_{3}\right)$ receptors. Eukaryotic cells have evolved a two-component store-operated $\mathrm{Ca}^{2+}$ entry (SOCE) pathway, which is mediated by the $\mathrm{Ca}^{2+}$ release activated $\mathrm{Ca}^{2+}$ (CRAC) channel, in response to depletion of $\mathrm{ER} \mathrm{Ca}^{2+}$ stores[1-5]. The first component of SOCE/CRAC is the $\mathrm{ER} \mathrm{Ca}^{2+}$ sensing proteins, stromal interacting molecules (STIM1 and STIM2) that change conformation upon ER $\mathrm{Ca}^{2+}$ depletion and translocate to ER-PM junctions [6,7]. Within these junctions, STIM proteins physically trap and gate ORAI proteins at the PM, the second component of SOCE representing the pore-forming subunits of CRAC channels[8-10]. SOCE has perhaps evolved first as means to replenish depleted ER $\mathrm{Ca}^{2+}$ but was coopted later during evolution for the purpose of cellular signaling that controls the induction of metabolic and transcriptional programs. CRAC channels are characterized by high $\mathrm{Ca}^{2+}$ selectivity with a reversal potential of $\sim+60 \mathrm{mV}$, depotentiation of $\mathrm{Na}^{+}$currents when recorded in divalent free solutions, small unitary conductance, conduction of $\mathrm{Sr}^{2+}$ and $\mathrm{Ba}^{2+}$ ions, and two modes of $\mathrm{Ca}^{2+}$-mediated negative feedback regulation in the form of fast (within milliseconds) and slow (within seconds to minutes) $\mathrm{Ca}^{2+}$-dependent inactivation (CDI)[3,11]. Interestingly, a portion of STIM1 C-terminus is essential for ORAI1 fast CDI[12,13], and $\mathrm{Ca}^{2+} /$ calmodulin binding to STIM1 appears to disrupt STIM1/ORAI1 interactions to mediate slow CDI $[14,15]$.

ORAI1 was discovered in genome-wide siRNA screens as an essential component of SOCE and CRAC channels[8-10]. ORAI2 and ORAI3 did not produce hits in those siRNA screens, but were identified by their significant sequence homology to ORAI1[8]. Each of the three mammalian ORAI isoforms can form functional CRAC channels when ectopically coexpressed with STIM1 in HEK293 cells[16,17]. However, while ORAI1 is clearly required for the function of native CRAC channels, the contribution of ORAI2 and ORAI3 has remained nebulous. Notwithstanding that mRNA of all three ORAI isoforms are ubiquitously co-expressed, it was reasonably proposed early after their discovery that ORAI2 and ORAI3 might encode CRAC channels in specialized cells from different tissues[17]. However, over a decade of studies suggested that this is largely not the case and the significance of ORAI2 and ORAI3 to native CRAC channel function have remained, until recently, elusive.

\section{ORAl1 is an essential pore-forming subunit of native CRAC channels}

In addition to the discovery of ORAI1 in unbiased genome-wide siRNA screens[8-10], several key findings cemented the position of ORAI1 as the bona fide pore-forming protein of CRAC channels and spawned a flurry of studies focused on ORAI1 in the past 15 years. These findings include: i) a point mutation in ORAI1 (R91W) that results in a severe combined immunodeficiency (SCID) syndrome due to lack of SOCE and CRAC currents in lymphocytes[8]. ii) Mutations within the putative pore region of ORAI1 generated either a dead CRAC channel or a channel with altered ion selectivity[18,19], and iii) the reconstitution of large CRAC currents by the ectopic co-expression of ORAI1 with STIM1[20-22]. The collective focus on ORAI1 resulted in the rapid identification of ORAI1 as a crucial component of SOCE in a growing number of primary cell types and identified mutations or altered expression of ORAI1 (and STIM1) as a contributor to several 
diseases[23-25]. Except for few cases discussed below, only ORAI1 was consistently revealed as critical for native CRAC channel function in a number of cell lines and primary cells from different tissue origins, with no discernible roles for ORAI2 and ORAI3. The resolution of the crystal structure of the sole Orai isoform in invertebrates, drosophila Orai (dOrai), at 3.35 angstrom revealed that six dOrai subunits are concentrically organized in a three-fold symmetry to form a channel pore with six glutamates (E106 in human ORAI1) on the extracellular side forming the selectivity filter[26]. Subsequent studies utilizing concatenated oligomers of human ORAI1 suggested that the human CRAC channel is likely a hexamer[27,28]. Nevertheless, a key question remained unanswered for the past 15 years: If ORAI1 alone is required for native CRAC channels and ORAI2 and ORAI3 are not universally encoding CRAC channels in primary cell types from different mammalian tissues and organs, what then is the signaling function of ORAI2 and ORAI3?

\section{Ectopically expressed ORAI2 and ORAI3 are structurally and functionally distinct from ORAl1}

Mammalian ORAI1 has the closest homology to the founding invertebrate member, drosophila Orai (dOrai). Evolutionarily, ORAI2 arose in vertebrates while ORAI3, the last ORAI isoform, appeared in mammals where the Orai3 gene is thought to have duplicated from Orai1[29]. While there is significant sequence homology between the pore region of all ORAI isoforms, their $\mathrm{N}$ - and C-termini are different, especially the C-terminus of ORAI3, which is significantly shorter compared to ORAI1 and ORAI2[30]. These structural differences likely contribute to the unique pharmacological profile of CRAC channels generated by ectopic co-expression of individual ORAI isoforms with STIM1. For example, while all three ectopically expressed ORAI isoforms are blocked by low concentrations of lanthanides $\left(1-5 \mu \mathrm{M} \mathrm{Gd}^{3+}\right)[31]$, the pharmacological compound 2-aminoethoxydiphenyl borate (2-APB) potentiates ORAI3 independently of store depletion while blocking ORAI1 and ORAI2[31-35]. A small molecule termed IA65 was discovered recently and shown to selectively potentiate ORAI1 with no significant effect on ORAI3[36] (Table 1). As mentioned above, side by side comparisons of ORAI1, ORAI2 and ORAI3 individually coexpressed with STIM1 in HEK293 cells showed that all three ORAI isoforms can support CRAC currents. However, ORAI1 supported the largest peak CRAC currents with ORAI2 and ORAI3 generating currents that were $\sim 50 \%$ and $\sim 20 \%$ the size of ORAI1, respectively[16,17,31]. These differences in peak currents between ORAI isoforms could be explained by differences in their fast CDI. ORAI3 showed the largest fast CDI, whereas ORAI1 showed the smallest with ORAI2 fast CDI falling in between[16,17]. Quite interestingly, Only ORAI1 is sensitive to slow CDI whereas ORAI2 and ORAI3 are insensitive[17] (Table 1).

\section{ORAI2 and ORAI3 heteromerize with ORAl1 to regulate CRAC channel function}

The first subtle hint that ORAI1 alone might not encompass CRAC channels came earlier from ectopic co-expression of ORAI1 with STIM1 in HEK293 cells. The large CRAC currents generated in these experiments recapitulated most, but not all, of the biophysical 
properties of native CRAC currents[21]. In ion substitution experiments, the $\mathrm{Sr}^{2+}$ and $\mathrm{Ba}^{2+}$ currents mediated by overexpressed STIM1 and ORAI1 were smaller than those typically observed for native CRAC channels[21], suggesting that some native regulatory factor or protein was missing from these overexpression experiments. A thorough study by Gwack et al. demonstrated using co-immunoprecipitations of tagged ectopically expressed proteins, that all ORAI isoforms physically interact[37]. FRET studies on ectopically expressed proteins also indicated that all three ORAI isoforms interact, and this interaction is not enhanced by ER store depletion[31]. While these reports indicated that overexpressed ORAI can heteromerize to form CRAC channels, the earliest evidence of a contribution of an ORAI isoform, other than ORAI1, to native CRAC was obtained from studies on ORAI1 knockout mice. The original report on ORAI1 knockout mice showed that mast cells from these mice have defective degranulation with a significant reduction of $~ 50 \%$ (but not a complete abrogation) of SOCE[38], suggesting a contribution of native ORAI2 and/or ORAI3 to the remaining SOCE. Surprisingly, and in stark contrast to human T-cells from SCID patients[8] or human cell lines[39], naïve T-cells from ORAI1 knockout mice showed very high mRNA expression of ORAI2 and unaltered proliferation, suggesting that, at least in the absence of ORAI1, ORAI2 can partially mediate native SOCE in mouse T-cells[38].

The first evidence that native ORAI2 and ORAI1 associate to form CRAC channels was provided by Vaeth et al in T-cells[40] and subsequently by Tsvilovskyy et al in mast cells[41]. Vaeth et al showed that SOCE in $\mathrm{CD}^{+} \mathrm{T}$ cells from T-cell specific ORAI1 knockout mice was partially reduced. Unexpectedly however, they discovered a significant increase in SOCE and CRAC currents in CD4 ${ }^{+} \mathrm{T}$ cells from global ORAI2 knockout mice, whereas the combination of ORAI2 and ORAI1 knockout in mouse T-cells abolished SOCE[40]. Tsvilovskyy et al reported increased SOCE in mast cells from ORAI2 knockout mice and this correlated with increased mast cell degranulation, core body temperature, and anaphylaxis[41]. These independent findings suggest that native ORAI1 and ORAI2 form heteromeric CRAC channels, where ORAI2 serves as a negative modulator of CRAC channel function[40,41]. Examination of dental enamel cells from ORAI2 knockout mice and dental enamel cell lines subjected to shRNA-mediated ORAI3 knockdown showed enhanced SOCE in the absence of ORAI2 or with knockdown of ORAI3[42], suggesting that ORAI2 and ORAI3 are negative regulators of SOCE. Indeed, a more recent side by side evaluation of the contribution of native ORAI isoforms to SOCE in HEK293 cells using CRISPR/Cas9-mediated single, double and triple Orai gene knockout revealed that both native ORAI2 and ORAI3 act as negative regulators of SOCE showing additive effects, with ORAI2 imparting larger negative regulation than ORAI3[31]. Together, these results support the notion that both ORAI2 and ORAI3 interact with ORAI1 to form native CRAC channels and serve as negative regulators that fine-tune CRAC channel activity.

However, studies in neurons paint a different picture for the role of ORAI2 in SOCE. Neurons from ORAI2 knockout mice have reduced SOCE and these mice are protected from stroke, presumably from reduced $\mathrm{Ca}^{2+}$-mediated neuronal death[43]. This positive participation of ORAI2 in neuronal SOCE is likely due to the high level of expression of ORAI2 in neurons, where only ORAI2 mRNA was detected while mRNAs for ORAI1 and ORAI3 were not[44]. Consistent with these results, neurons from STIM2 knockout mice have reduced SOCE while those from STIM1 knockout and ORAI1 knockout mice have 
unaltered SOCE [45], suggesting that neurons might have coopted the STIM2/ORAI2 pair for SOCE. Just like the case of ORAI2 in neurons, the first evidence that ORAI3 contributes to native SOCE was obtained from a subset of estrogen receptor positive breast cancer cell lines, which show high levels of expression of ORAI3[46,47].

Furthermore, potential interactions between native ORAI1 and ORAI3 were inferred from the high sensitivity to $\mathrm{H}_{2} \mathrm{O}_{2}$-mediated inhibition of SOCE in naïve $\mathrm{CD} 4^{+} \mathrm{T}$ cells and monocytes compared to differentiated cells. The enhanced resistance to $\mathrm{H}_{2} \mathrm{O}_{2}$ correlated with an increase in ORAI3 expression[48], as the redox-resistant ORAI3 lacks the equivalent of cysteine-195 that mediates $\mathrm{H}_{2} \mathrm{O}_{2}$ inactivation of ORAI1[49] (Table 1). Increased ORAI3 expression is believed to alter the composition of CRAC channels generating more redox-resistant channels and it seems that the presence of one ORAI3 subunit in a concatenated tetramer was sufficient to protect CRAC channels from oxidative inactivation[50]. Therefore, it appears that under certain conditions native ORAI2 and ORAI3 can function independently to mediate SOCE and that ORAI3 can serve as a positive modulator of SOCE in redox-rich environments where ORAI3 protects against redoxmediated SOCE inhibition.

\section{Native CRAC channels: dynamic heteromers of all ORAls tailored to diverse $\mathrm{Ca}^{2+}$ signaling events}

As it turned out, the negative regulation of CRAC channels by heteromerization of ORAI2 and/or ORAI3 with ORAI1 reveals only a portion of the full picture. In fact, most studies to date (discussed above) used protocols that cause maximal store depletion to generate supramaximal $\mathrm{Ca}^{2+}$ signals that typically manifest as a sustained cytosolic $\mathrm{Ca}^{2+}$ plateau[51]. Physiological $\mathrm{Ca}^{2+}$ signaling events in vivo are seldom of this large magnitude, except perhaps during cell death and primary cells in vivo tend to respond to very low concentrations of agonists compared to cultured cells[52]. Thus, physiological $\mathrm{Ca}^{2+}$ signals encompass a large repertoire of oscillatory $\mathrm{Ca}^{2+}$ events or spikes with diverse amplitudes and frequencies that increase with increasing concentrations of the stimulus and only manifest as plateaus with maximal agonist stimulation. With this in mind, CRISPR/Cas9mediated knockout combinations of ORAI isoforms showed that native ORAI3 and ORAI2 can support regenerative $\mathrm{Ca}^{2+}$ oscillations but only ORAI1 supported $\mathrm{Ca}^{2+}$ plateaus. This behavior may be due to the strong basal interactions of ORAI2 and ORAI3 with STIM1 and the heightened fast $\mathrm{Ca}^{2+}$-dependent inactivation (CDI) of these two isoforms $[31,53]$. The preferential basal interaction of STIM1 with ORAI2 and ORAI3 over ORAI1 could potentially allow CRAC channels consisting of ORAI 2 and/or ORAI3 to be rapidly activated and inactivated to suit the small and pulsatile oscillatory $\mathrm{Ca}^{2+}$ signaling in response to low and moderate physiological agonist stimulation. Of note, ORAI1/ORAI3 heteromeric assemblies involving basal interactions of ORAI3 with STIM1 were proposed to mediate a $\mathrm{Ca}^{2+}$ entry route activated independently of store depletion at low physiological concentrations of agonist and involving, through unknown mechanisms, arachidonic acid or its metabolite leukotrience $\mathrm{C}_{4}$ [54-57]. Whether this store-independent pathway is simply a variant of SOCE that is activated by low concentrations of agonists that might not achieve measurable ER depletion is an interesting question that requires further investigations. 
These results imply that native CRAC channels either have a dynamic stoichiometry or present with a multitude of fixed stoichiometries, presumably depending on the relative expression levels of each of the three ORAI isoforms within a specific cell type.

Heteromeric CRAC channels with higher proportions of ORAI2 and ORAI3 are expected to be gated by STIM1 at low agonist concentrations than those consisting predominantly of ORAI1. These heteromeric CRAC channels would generate a tempered SOCE activity and NFAT activation that is tailored to the strength of agonist stimulation. In fact, deletion of native ORAI2 and ORAI3 in HEK293 cells changed the cell oscillatory $\mathrm{Ca}^{2+}$ signal in response to low and moderate agonist concentrations into mostly $\mathrm{Ca}^{2+}$ plateaus and generated maximal NFAT4 and NFAT1 isoform nuclear translocation, essentially restricting the graded diversity of agonist-activated $\mathrm{Ca}^{2+}$ signals and the differential induction of NFAT isoforms normally activated by distinct magnitudes of $\mathrm{Ca}^{2+}$ signals[31]. The graded diversity of $\mathrm{Ca}^{2+}$ signals would be analogous to the graded intensity of light emitted by a dimmable electrical bulb. With ORAI1 alone the system is restricted to an On/Off switch while addition of ORAI2 and ORAI3 endows the system with the fine control of a dimmer switch.

\section{Conclusions}

Currently, there is no structural or proteomic evidence shedding light on the stoichiometry or stoichiometries of CRAC channels in native tissues or data ruling out the coexistence of certain stoichiometries, including homomers of each ORAI isoform. Nevertheless, knockout models and data on $\mathrm{Ca}^{2+}$ oscillations mediated by native SOCE in HEK293 cells cannot be reconciled with the exclusive presence of ORAI isoform homomers[31]. Rather, these data support the idea that ORAI isoforms heteromerize to form native CRAC channels (Figure 1). This heteromerization ensures the graded diversity of CRAC-mediated $\mathrm{Ca}^{2+}$ signaling events in response to the full range of agonist strengths and the specific activation of a given transcription factor isoform (e.g. NFAT) at its specific range of cytosolic $\mathrm{Ca}^{2+}$ concentration. The presence of ORAI2 and ORAI3 within the native CRAC channel ensures the differential activation of NFAT4 (requiring a low $\mathrm{Ca}^{2+}$ signal) and NFAT1 (requiring a high $\mathrm{Ca}^{2+}$ signal) isoforms at distinct agonist concentrations. With CRAC channels containing ORAI1 alone, the $\mathrm{Ca}^{2+}$ signal is already maximal at lower concentrations of agonist leading to mostly concomitant NFAT4 and NFAT1 nuclear translocation[31]. Obviously, it is difficult to currently predict if there is only one native CRAC channel species with a specific fixed ORAI isoform stoichiometry or a dynamic one, or that many ORAI combinations generate a wide variety of native CRAC channels. The latter situation is more likely and the diversity of native CRAC channels in any given cell type would likely depend on the relative expression of each ORAI isoform in these cells. At this stage, the presence of functional homomeric CRAC channels composed of one native ORAI isoform cannot be ruled out (Figure 1). Because native ORAI2 and ORAI3 either alone or together are not capable of supporting NFAT isoform nuclear translocation in HEK293 cells[31], CRAC channels composed exclusively of ORAI2 and/or ORAI3, if they widely exist, likely signal to different downstream pathways and transcription factors. Similarly, whether native CRAC channels are predominantly heteromers of two or three ORAI isoforms or both remain unclear. The choreography of native CRAC channels becomes even more complex when we consider the 
dynamic and privileged interactions of STIM1 and STIM2 with individual ORAIs during CRAC channel activation at the full range of physiological agonist stimulation[58,59]. Furthermore, relative contributions of STIM1 and STIM2 are likely dependent on the levels of expression of their splice variants and their potential regulation by posttranslational modifications in a given cell type. For instance, in monocytes, neurons, and colorectal cancer cells, knockdown or knockout of STIM2 has a profound effect on SOCE, causing a reduction by $>50 \%$, whereas STIM2 knockout in many cell types including HEK293 cells reduces SOCE by only $\sim 10-15 \%[39,45,48,60]$. Currently, important tools such as reliable antibodies capable of specifically detecting endogenous levels of ORAI2 and ORAI3 are missing. Further, the crystal structures of mammalian ORAI1, ORAI2 and ORAI3 have yet to be resolved. These structures and those of STIM/ORAI interactions would provide critical insights into the molecular composition of the native CRAC channel in various primary cell types, and these insights would be of crucial importance to specific targeting of this important $\mathrm{Ca}^{2+}$ entry route for the purpose of disease therapy.

\section{Acknowledgements}

Due to space restrictions, we apologize to the many colleagues we did not cite. In particular, we sincerely apologize to colleagues, whose work is relevant to the topic of native CRAC channel makeup, we inadvertently failed to cite. Work in our laboratory is supported by the National Heart, Lung, and Blood Institute R35-HL150778 to M.T.

\section{References:}

1. Trebak M, Kinet JP: Calcium signalling in T cells. Nat Rev Immunol 2019, 19:154-169. [PubMed: 30622345] (*) A recent review on $\mathrm{Ca}^{2+}$ signaling in T-cells, which discusses the emerging functions of ORAI proteins

2. Putney JW Jr.: A model for receptor-regulated calcium entry. Cell Calcium 1986, 7:1-12. [PubMed: 2420465]

3. Prakriya M, Lewis RS: Store-Operated Calcium Channels. Physiol Rev 2015, 95:1383-1436. [PubMed: 26400989]

4. Lewis RS: Store-Operated Calcium Channels: From Function to Structure and Back Again. Cold Spring Harb Perspect Biol 2020, 12.

5. Lunz V, Romanin C, Frischauf I: STIM1 activation of Orai1. Cell Calcium 2019, 77:29-38. [PubMed: 30530091]

6. Liou J, Kim ML, Heo WD, Jones JT, Myers JW, Ferrell JE Jr., Meyer T: STIM is a Ca2+ sensor essential for Ca2+-store-depletion-triggered Ca2+ influx. Curr Biol 2005, 15:1235-1241. [PubMed: 16005298]

7. Roos J, DiGregorio PJ, Yeromin AV, Ohlsen K, Lioudyno M, Zhang S, Safrina O, Kozak JA, Wagner SL, Cahalan MD, et al.: STIM1, an essential and conserved component of store-operated Ca2+ channel function. J Cell Biol 2005, 169:435-445. [PubMed: 15866891]

8. Feske S, Gwack Y, Prakriya M, Srikanth S, Puppel SH, Tanasa B, Hogan PG, Lewis RS, Daly M, Rao A: A mutation in Orail causes immune deficiency by abrogating CRAC channel function. Nature 2006, 441:179-185. [PubMed: 16582901]

9. Vig M, Peinelt C, Beck A, Koomoa DL, Rabah D, Koblan-Huberson M, Kraft S, Turner H, Fleig A, Penner R, et al.: CRACM1 is a plasma membrane protein essential for store-operated Ca2+ entry. Science 2006, 312:1220-1223. [PubMed: 16645049]

10. Zhang SL, Yeromin AV, Zhang XH, Yu Y, Safrina O, Penna A, Roos J, Stauderman KA, Cahalan MD: Genome-wide RNAi screen of $\mathrm{Ca}(2+)$ influx identifies genes that regulate $\mathrm{Ca}(2+)$ releaseactivated $\mathrm{Ca}(2+)$ channel activity. Proc Natl Acad Sci U S A 2006, 103:9357-9362. [PubMed: 16751269] 
11. Krizova A, Maltan L, Derler I: Critical parameters maintaining authentic CRAC channel hallmarks. Eur Biophys J 2019, 48:425-445. [PubMed: 30903264]

12. Lee KP, Yuan JP, Zeng W, So I, Worley PF, Muallem S: Molecular determinants of fast Ca2+dependent inactivation and gating of the Orai channels. Proc Natl Acad Sci U S A 2009, 106:14687-14692. [PubMed: 19706554]

13. Mullins FM, Lewis RS: The inactivation domain of STIM1 is functionally coupled with the Orai1 pore to enable Ca2+-dependent inactivation. Journal of General Physiology 2016, 147:153-164. [PubMed: 26809794]

14. Bhardwaj R, Augustynek BS, Ercan-Herbst E, Kandasamy P, Seedorf M, Peinelt C, Hediger MA: $\mathrm{Ca}(2+) / C a l m o d u l i n$ Binding to STIM1 Hydrophobic Residues Facilitates Slow Ca(2+)-Dependent Inactivation of the Orai1 Channel. Cell Physiol Biochem 2020, 54:252-270. [PubMed: 32176842]

15. Li X, Wu G, Yang Y, Fu S, Liu X, Kang H, Yang X, Su XC, Shen Y: Calmodulin dissociates the STIM1-Orai1 complex and STIM1 oligomers. Nat Commun 2017, 8:1042. [PubMed: 29051492]

16. DeHaven WI, Smyth JT, Boyles RR, Putney JW Jr.: Calcium inhibition and calcium potentiation of Orai1, Orai2, and Orai3 calcium release-activated calcium channels. J Biol Chem 2007, 282:17548-17556. [PubMed: 17452328]

17. Lis A, Peinelt C, Beck A, Parvez S, Monteilh-Zoller M, Fleig A, Penner R: CRACM1, CRACM2, and CRACM3 are store-operated $\mathrm{Ca} 2+$ channels with distinct functional properties. Curr Biol 2007, 17:794-800. [PubMed: 17442569]

18. Prakriya M, Feske S, Gwack Y, Srikanth S, Rao A, Hogan PG: Orai1 is an essential pore subunit of the CRAC channel. Nature 2006, 443:230-233. [PubMed: 16921383]

19. Vig M, Beck A, Billingsley JM, Lis A, Parvez S, Peinelt C, Koomoa DL, Soboloff J, Gill DL, Fleig A, et al.: CRACM1 multimers form the ion-selective pore of the CRAC channel. Current biology : CB 2006, 16:2073-2079. [PubMed: 16978865]

20. Mercer JC, Dehaven WI, Smyth JT, Wedel B, Boyles RR, Bird GS, Putney JW Jr.: Large storeoperated calcium selective currents due to co-expression of Orai1 or Orai2 with the intracellular calcium sensor, Stim1. J Biol Chem 2006, 281:24979-24990. [PubMed: 16807233]

21. Peinelt C, Vig M, Koomoa DL, Beck A, Nadler MJ, Koblan-Huberson M, Lis A, Fleig A, Penner R, Kinet JP: Amplification of CRAC current by STIM1 and CRACM1 (Orai1). Nat Cell Biol 2006, 8:771-773. [PubMed: 16733527]

22. Soboloff J, Spassova MA, Tang XD, Hewavitharana T, Xu W, Gill DL: Orai1 and STIM reconstitute store-operated calcium channel function. J Biol Chem 2006, 281:20661-20665. [PubMed: 16766533]

23. Johnson M, Trebak M: ORAI channels in cellular remodeling of cardiorespiratory disease. Cell Calcium 2019, 79:1-10. [PubMed: 30772685]

24. Lacruz RS, Feske S: Diseases caused by mutations in ORAI1 and STIM1. Ann N Y Acad Sci 2015, 1356:45-79. [PubMed: 26469693]

25. Vashisht A, Trebak M, Motiani RK: STIM and Orai proteins as novel targets for cancer therapy. A Review in the Theme: Cell and Molecular Processes in Cancer Metastasis. Am J Physiol Cell Physiol 2015, 309:C457-469. [PubMed: 26017146]

26. Hou X, Pedi L, Diver MM, Long SB: Crystal structure of the calcium release-activated calcium channel Orai. Science 2012, 338:1308-1313. [PubMed: 23180775]

27. Cai X, Zhou Y, Nwokonko RM, Loktionova NA, Wang X, Xin P, Trebak M, Wang Y, Gill DL: The Orai1 Store-operated Calcium Channel Functions as a Hexamer. J Biol Chem 2016, 291:2576425775. [PubMed: 27780862]

28. Yen M, Lokteva LA, Lewis RS: Functional Analysis of Orai1 Concatemers Supports a Hexameric Stoichiometry for the CRAC Channel. Biophys J 2016, 111:1897-1907. [PubMed: 27806271]

29. Cai X: Molecular Evolution and Structural Analysis of the Ca2+ Release-Activated Ca2+ Channel Subunit, Orai. Journal of Molecular Biology 2007, 368:1284-1291. [PubMed: 17400243]

30. Shuttleworth TJ: Orai3--the 'exceptional' Orai? The Journal of physiology 2012, 590:241-257. [PubMed: 22041188]

31. Yoast RE, Emrich SM, Zhang X, Xin P, Johnson MT, Fike AJ, Walter V, Hempel N, Yule DI, Sneyd J, et al.: The native ORAI channel trio underlies the diversity of $\mathrm{Ca} 2+$ signaling events. Nature Communications 2020, 11.(**) The first evidence that all three ORAI isoforms 
heteromerize to form CRAC channels that mediate the graded diversity of $\mathrm{Ca}^{2+}$ signaling events in response to the full range of agonist concentrations.

32. Kappel S, Kilch T, Baur R, Lochner M, Peinelt C: The Number and Position of Orai3 Units within Heteromeric Store-Operated $\mathrm{Ca}(2+)$ Channels Alter the Pharmacology of ICRAC. Int J Mol Sci 2020, 21.

33. Schindl R, Bergsmann J, Frischauf I, Derler I, Fahrner M, Muik M, Fritsch R, Groschner K, Romanin C: 2-aminoethoxydiphenyl borate alters selectivity of Orai3 channels by increasing their pore size. J Biol Chem 2008, 283:20261-20267. [PubMed: 18499656]

34. Peinelt C, Lis A, Beck A, Fleig A, Penner R: 2-Aminoethoxydiphenyl borate directly facilitates and indirectly inhibits STIM1-dependent gating of CRAC channels. The Journal of physiology 2008, 586:3061-3073. [PubMed: 18403424]

35. DeHaven WI, Smyth JT, Boyles RR, Bird GS, Putney JW Jr.: Complex actions of 2aminoethyldiphenyl borate on store-operated calcium entry. The Journal of biological chemistry 2008, 283:19265-19273. [PubMed: 18487204]

36. Azimi I, Stevenson RJ, Zhang X, Meizoso-Huesca A, Xin P, Johnson M, Flanagan JU, Chalmers $\mathrm{SB}$, Yoast RE, Kapure JS, et al.: A new selective pharmacological enhancer of the Orai1 $\mathrm{Ca}(2+)$ channel reveals roles for Orai1 in smooth and skeletal muscle functions. ACS pharmacology \& translational science 2020, 3:135-147. [PubMed: 32190822]

37. Gwack Y, Srikanth S, Feske S, Cruz-Guilloty F, Oh-hora M, Neems DS, Hogan PG, Rao A: Biochemical and functional characterization of Orai proteins. J Biol Chem 2007, 282:1623216243. [PubMed: 17293345]

38. Vig M, DeHaven WI, Bird GS, Billingsley JM, Wang H, Rao PE, Hutchings AB, Jouvin MH, Putney JW, Kinet JP: Defective mast cell effector functions in mice lacking the CRACM1 pore subunit of store-operated calcium release-activated calcium channels. Nat Immunol 2008, 9:89-96. [PubMed: 18059270]

39. Zhang X, Pathak T, Yoast R, Emrich S, Xin P, Nwokonko RM, Johnson M, Wu S, Delierneux C, Gueguinou M, et al.: A calcium/cAMP signaling loop at the ORAI1 mouth drives channel inactivation to shape NFAT induction. Nature Communications 2019, 10:1971.

40. Vaeth M, Yang J, Yamashita M, Zee I, Eckstein M, Knosp C, Kaufmann U, Karoly Jani P, Lacruz RS, Flockerzi V, et al.: ORAI2 modulates store-operated calcium entry and T cell-mediated immunity. Nature communications 2017, 8:14714-14714.(**)The first to use T cells from ORAI2 knockout mice and from ORAI1/ORAI2 double knockout mice to show that ORAI2 hetermerizes with ORAI1 to negatively regulate CRAC channel function.

41. Tsvilovskyy V, Solis-Lopez A, Schumacher D, Medert R, Roers A, Kriebs U, Freichel M: Deletion of Orai2 augments endogenous CRAC currents and degranulation in mast cells leading to enhanced anaphylaxis. Cell Calcium 2018, 71:24-33. [PubMed: 29604961] (*) Along with \#40, this study used mast cells from ORAI2 knockout mice to demonstrate that ORAI2 is a negative regulator of CRAC channels.

42. Eckstein M, Vaeth M, Aulestia FJ, Costiniti V, Kassam SN, Bromage TG, Pedersen P, Issekutz T, Idaghdour Y, Moursi AM, et al.: Differential regulation of $\mathrm{Ca}^{2+}$ influx by ORAI channels mediates enamel mineralization. Science Signaling 2019, 12:eaav4663. [PubMed: 31015290] (*) This excellent study used gene deletion or knockdown of all ORAI isoforms to demonstrate that ORAI2 and ORAI3 serve as negative regulators of SOCE in enamel cells.

43. Stegner D, Hofmann S, Schuhmann Michael K, Kraft P, Herrmann Alexander M, Popp S, Höhn M, Popp M, Klaus V, Post A, et al.: Loss of Orai2-Mediated Capacitative Ca2+ Entry Is Neuroprotective in Acute Ischemic Stroke. Stroke 2019, 50:3238-3245. [PubMed: 31551038]

44. Chauvet S, Jarvis L, Chevallet M, Shrestha N, Groschner K, Bouron A: Pharmacological Characterization of the Native Store-Operated Calcium Channels of Cortical Neurons from Embryonic Mouse Brain. Frontiers in pharmacology 2016, 7:486-486. [PubMed: 28018223]

45. Berna-Erro A, Braun A, Kraft R, Kleinschnitz C, Schuhmann MK, Stegner D, Wultsch T, Eilers J, Meuth SG, Stoll G, et al.: STIM2 regulates capacitive Ca2+ entry in neurons and plays a key role in hypoxic neuronal cell death. Sci Signal 2009, 2:ra67. [PubMed: 19843959]

46. Motiani RK, Abdullaev IF, Trebak M: A novel native store-operated calcium channel encoded by Orai3: selective requirement of Orai3 versus Orai1 in estrogen receptor-positive versus estrogen 
receptor-negative breast cancer cells. The Journal of biological chemistry 2010, 285:19173-19183. [PubMed: 20395295]

47. Motiani RK, Zhang X, Harmon KE, Keller RS, Matrougui K, Bennett JA, Trebak M: Orai3 is an estrogen receptor a-regulated $\mathrm{Ca}^{2+}$ channel that promotes tumorigenesis. FASEB journal : official publication of the Federation of American Societies for Experimental Biology 2013, 27:63-75. [PubMed: 22993197]

48. Saul S, Gibhardt C, Schmidt B, Lis A, Pasieka B, Conrad D, Jung P, Gaupp R, Wonnenberg B, Diler E, et al.: A calcium-redox feedback loop controls human monocyte immune responses: The role of ORAI Ca2+ channels. Science Signaling 2016, 9:ra26-ra26. [PubMed: 26956485]

49. Bogeski I, Kummerow C, Al-Ansary D, Schwarz EC, Koehler R, Kozai D, Takahashi N, Peinelt C, Griesemer D, Bozem M, et al.: Differential redox regulation of ORAI ion channels: a mechanism to tune cellular calcium signaling. Sci Signal 2010, 3:ra24. [PubMed: 20354224]

50. Alansary D, Bogeski I, Niemeyer BA: Facilitation of Orai3 targeting and store-operated function by Orai1. Biochim Biophys Acta 2015, 1853:1541-1550. [PubMed: 25791427]

51. Putney JW: Calcium signaling: deciphering the calcium-NFAT pathway. Curr Biol 2012, 22:R8789. [PubMed: 22321308]

52. McCarron JG, Wilson C, Heathcote HR, Zhang X, Buckley C, Lee MD: Heterogeneity and emergent behaviour in the vascular endothelium. Curr Opin Pharmacol 2019, 45:23-32. [PubMed: 31005824]

53. Zhang X, Gonzalez-Cobos JC, Schindl R, Muik M, Ruhle B, Motiani RK, Bisaillon JM, Zhang W, Fahrner M, Barroso M, et al.: Mechanisms of STIM1 activation of store-independent leukotriene C4-regulated Ca2+ channels. Mol Cell Biol 2013, 33:3715-3723. [PubMed: 23878392]

54. Gonzalez-Cobos JC, Zhang X, Zhang W, Ruhle B, Motiani RK, Schindl R, Muik M, Spinelli AM, Bisaillon JM, Shinde AV, et al.: Store-independent Orai1/3 channels activated by intracrine leukotriene C4: role in neointimal hyperplasia. Circ Res 2013, 112:1013-1025. [PubMed: 23349245]

55. Shuttleworth TJ, Mignen O: Calcium entry and the control of calcium oscillations. Biochemical Society Transactions 2003, 31:916-919. [PubMed: 14505448]

56. Thompson JL, Shuttleworth TJ: Molecular basis of activation of the arachidonate-regulated $\mathrm{Ca} 2+$ (ARC) channel, a store-independent Orai channel, by plasma membrane STIM1. J Physiol 2013, 591:3507-3523. [PubMed: 23690558]

57. Zhang X, Zhang W, Gonzalez-Cobos JC, Jardin I, Romanin C, Matrougui K, Trebak M: Complex role of STIM1 in the activation of store-independent Orai1/3 channels. J Gen Physiol 2014, 143:345-359. [PubMed: 24567509]

58. Ong HL, de Souza LB, Zheng C, Cheng KT, Liu X, Goldsmith CM, Feske S, Ambudkar IS: STIM2 enhances receptor-stimulated $\mathrm{Ca}(2)(+)$ signaling by promoting recruitment of STIM1 to the endoplasmic reticulum-plasma membrane junctions. Sci Signal 2015, 8:ra3. [PubMed: 25587190]

59. Subedi KP, Ong HL, Son GY, Liu X, Ambudkar IS: STIM2 Induces Activated Conformation of STIM1 to Control Orai1 Function in ER-PM Junctions. Cell Rep 2018, 23:522-534. [PubMed: 29642009] (*)This study demonstrated the intertwinded functions of STIM1 and STIM 2 during SOCE, whereby STIM2 traps STIM1 and enhances its interaction with ORAI1 under conditions of moderate store depletion.

60. Emrich SM, Yoast RE, Xin P, Zhang X, Pathak T, Nwokonko R, Gueguinou M, Subedi KP, Zhou Y, Ambudkar IS, et al.: Crosstalk between N-terminal and C-terminal domains in stromal interaction molecule 2 (STIM2) determines enhanced STIM2 sensitivity. Journal of Biological Chemistry 2019:jbc.RA118.00680. 


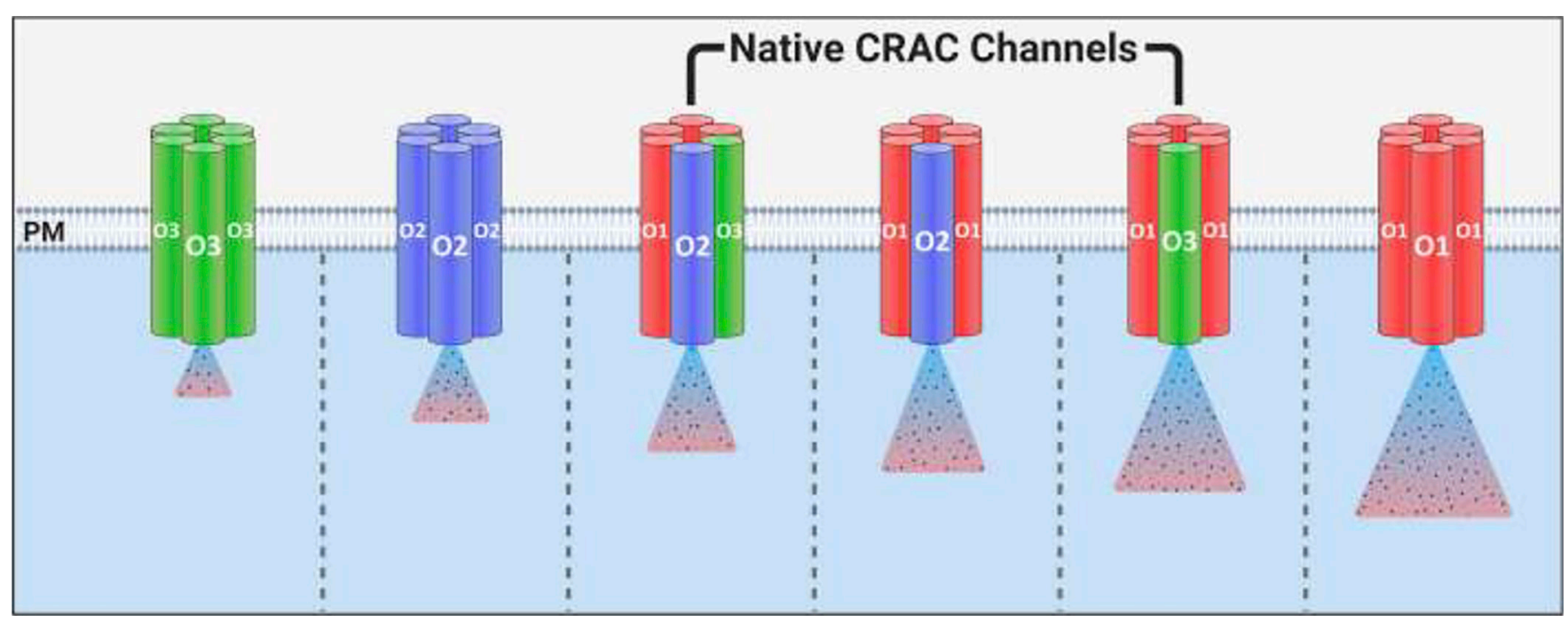

Figure 1. Native CRAC channels.

Native CRAC channels are likely heterohexamers of all three ORAI isoforms but their precise stoichiometry or stoichiometries remain unclear. The incorporation of ORAI2 and/or ORAI3 within a hexamer negatively regulates ORAI1 and fine tunes CRAC-mediated $\mathrm{Ca}^{2+}$ signaling. Whether native CRAC channels can also exist as homohexamers of each ORAI isoform remains an open question. 


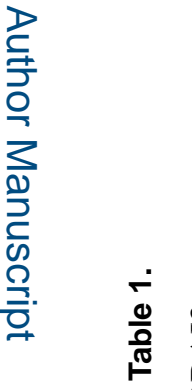

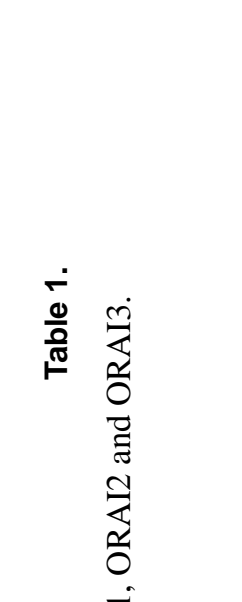

更

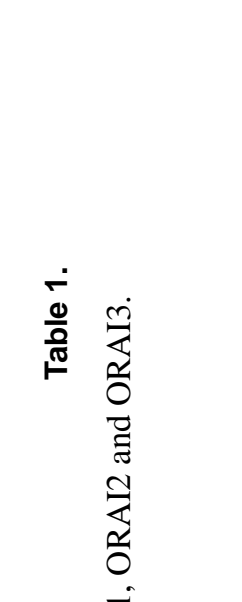

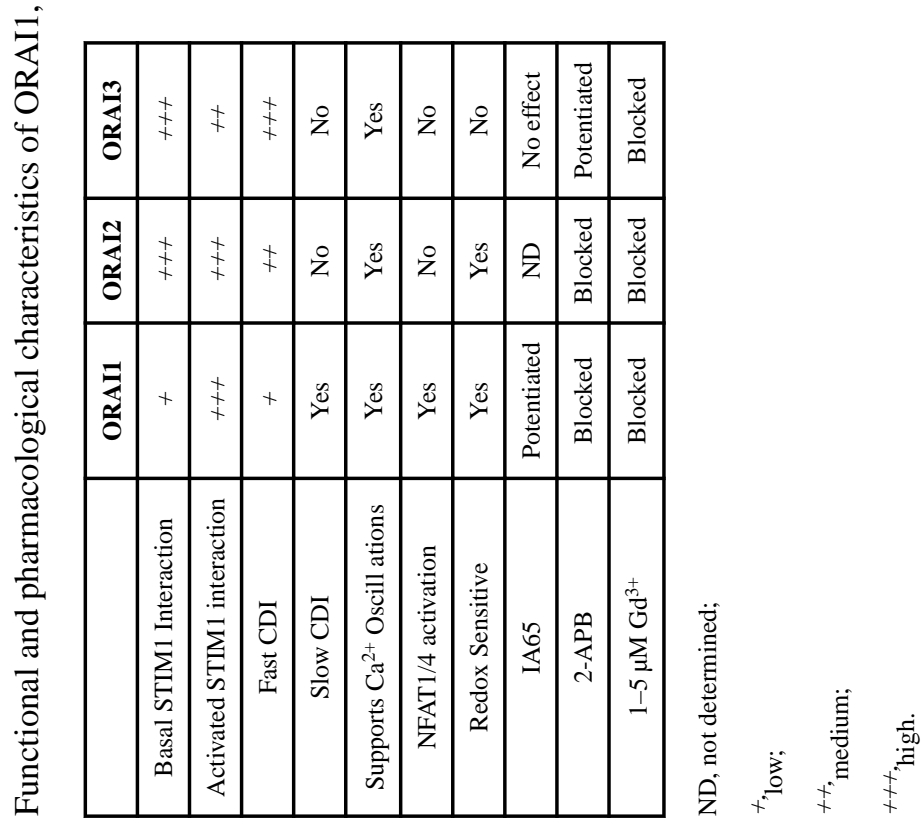

롤

Curr Opin Physiol. Author manuscript; available in PMC 2021 October 01. 